\title{
Anthrax Laboratory Diagnostic Methods at the Laboratory of the Ministry of Agriculture (LMA)
}

\section{Irma Beradze}

Animal Disease Diagnostics, LMA, Tbilisi, Georgia

Objective

Laboratory of the Ministry of Agriculture (LMA) conducts Anthrax diagnostics using Bacteriology and Molecular Biology Methods: Isolated cultures through the classical bacteriology methods are always confirmed by Molecular Biology assay (PCR).

In the study the samples were screened for the presence of $B$. anthracis via two concurrent approaches to compare classical methods and a novel PCR method. Before the TAP-7 project, PCR was only used to confirm the identity of cultures isolated by the Bacteriology. New SOPs and algorythm was created for better laboratory diagnostic.

\section{Introduction}

Bacillus anthracis, the etiologic agent of anthrax, is a member of a highly diverse group of endospore-forming bacteria. Bacillus anthracis spores are typically found in soil, from which they may spread via contaminated dust, water, and materials of plant and animal origin. Although anthrax is primarily a disease of herbivores, humans may contract anthrax directly or indirectly from animals.

Laboratory of the Ministry of Agriculture (LMA) conducts Anthrax diagnostics using Bacteriology and Molecular Biology Methods: Isolated cultures through the classical bacteriology methods are always confirmed by Molecular Biology assay (PCR).

In 2014, within Tap7 project, Identification and Mapping of Anthrax foci in Georgia' Anthrax suspected soil samples were tested using two lab diagnostic methods and they were compared to each other.

\section{Methods}

Anthrax suspected samples were tested by two methods - classical method and new method. Classical method included isolation of bacterium from soil samples using standard bacteriology tests and then PCR confirmed its identity. New method was initial PCR testing of soil samples 302 soil samples were tested by classical method. At the same time, approximately $10 \%$ ( 32 samples) of the already mentioned 302 soil samples were also tested by initial PCR.

\section{Results}

24 cultures isolated through bacteriology tests (Gram staining; lysis by gamma phage; motility testing; detection of polyDglutamic acid capsule by direct fluorescent antibody (DFA) were confirmed by PCR.

Out of the above mentioned 32-suspected samples, 11 were confirmed positive using the classical methods, versus 9 confirmed positive using the direct PCR approach. Two bacteriologically positive samples appeared negative by the direct PCR method, i.e. only two samples did not match.

\section{Conclusions}

The samples were screened for the presence of $B$. anthracis via two concurrent approaches to compare classical methods and a novel PCR method. Before the TAP-7 project, PCR was only used to confirm the identity of cultures isolated by the Bacteriology.

The purpose of the investigation of the new method was to identify if a less labor-intensive process with fewer points of operator manipulation was as efficacious as the classical method of bacteriology followed by PCR analysis of suspected samples.

Despise the limited sampling and the little difference in the efficacy of the two methods, classical method stays prior to new one. 